\title{
O I 22 Prevalence of drug interactions between antiretroviral and co-administered drugs at the Moi teaching and referral hospital (Ampath), Eldoret, Kenya
}

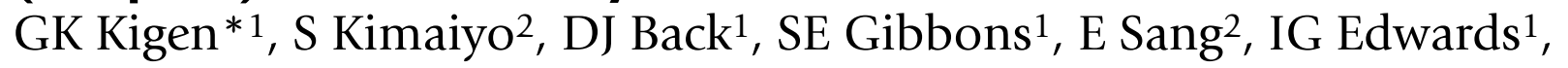
A Owen ${ }^{1}$ and $\mathrm{SH} \mathrm{Khoo}^{1}$

Address: ${ }^{1}$ University of Liverpool, Department of Pharmacology and Therapeutics, Liverpool, UK and ${ }^{2}$ Moi University School of Medicine, AMPATH, Eldoret, Kenya

* Corresponding author

from Ninth International Congress on Drug Therapy in HIV Infection

Glasgow, UK. 9-13 November 2008

Published: 10 November 2008

Journal of the International AIDS Society 2008, I I (SuppI I):O7 doi:I0.II86/I758-2652-II-SI-O7

This abstract is available from: http://www.jiasociety.org/content/II/SI/O7

(c) 2008 Kigen et al; licensee BioMed Central Ltd.

\section{Background}

Studies in developed countries have shown that drug interactions (DI) involving antiretroviral therapy (ARV) are frequent and under-recognized. No similar work has been carried out in developing countries, where use of fixed dose combinations (FDCs) limits the scope for dose modification.

\section{Methods}

Retrospective survey of a large database of HIV+ adults enrolled into Ampath programme. Details of age, gender, weight, ARVs and concomitant medications were recorded. DI were identified from http://www.hiv-drugin teractions.org and classified as: major (potential to cause life-threatening illness or hospitalization, 'contra-indicated' or 'not recommended' in SPC, or requiring dose modification of either, or both drugs); moderate (recommendation to 'avoid' in SPC, or having a $>50 \%$ effect on the AUC); minor (likelihood of interaction [actual or theoretical] or having an effect of $25-50 \%$ AUC).

\section{Summary of results}

Data from 1,000 consecutive patients (363 male, 637 female, aged 13 - 68 years; mean body weight $61.3 \mathrm{~kg}$ ), who were followed up for 1-21 months (median 9, total 8,594 patient-months of follow up) were included. 750 (75\%) were on first-line ARV ( $\mathrm{d} 4 \mathrm{~T}, 3 \mathrm{TC}, \mathrm{NVP})$ and the use of individual drugs was as follows: d4T 758(75.8\%), 3TC
950(95\%), ZDV 211(21.2\%), NVP 613 (61.3\%), EFV 264 (26.4\%), and LPVr 55 (5.5\%). All ARVs were prescribed at standard doses, regardless of whether a DI was present or not. Major interactions were identified in 187 patients $(18.7 \%)$, predominantly involving rifampicin 104 $(10.4 \%)$ and azoles 82 (8.2\%). Moderate interactions were identified in 142 patients $(14.2 \%)$, involving steroids $76(7.6 \%)$, azoles $34(3.4 \%)$ and antimalarials 27 $(2.7 \%)$ patients. Minor interactions were recorded for 44 patients (4.4\%). A total of 279 patients (27.9\%) had either a major or moderate interaction, while no drug interactions were recorded in 698 patients (69.8\%).

\section{Conclusion}

Clinically significant DIs were common, affecting over $1 /$ 4 patients receiving ART in Kenya, yet are frequently not recognized. Although TB medications accounted for a significant proportion of DIs, we identified other important interactions involving ARVs and azoles, as well as antimalarials. Given the relative lack of laboratory monitoring and widespread use of FDCs, strategies need to be developed urgently to avoid important drug interactions, to identify early markers of toxicity, and to manage unavoidable interactions safely, in order to reduce risk of harm, and to maximize the effectiveness of mass ARV deployment in Africa. 